\title{
COMPLETELY DISTRIBUTIVE COMPLETE LATTICES
}

\author{
GEORGE N. RANEY
}

1. Introduction. This paper is concerned with the representation theory for completely distributive complete lattices. The results obtained are extensions of similar results of Tarski on distributive lattices and on completely distributive Boolean algebras. ${ }^{1}$ The following definitions and notations are used.

If $F$ is a family of subsets of a set, the intersection of $F$ is denoted by $\Pi F$, and the union of $F$ is denoted by $\sum F$.

Definition 1. A family $R$ of subsets of a set is called a complete ring of sets if for every $F \subset R, \prod F \in R$ and $\sum F \in R$.

If $L$ is a complete lattice, then every subset $K$ of $L$ has a meet, which is denoted by $\cap K$, and a join, which is denoted by $U K$.

Definition 2. Let $L$ and $L^{\prime}$ be complete lattices. A mapping $\theta$ of $L$ into $L^{\prime}$ is called a complete homomorphism if for every subset $K$ of $L$, $\theta(\cap K)=\cap \theta(K)$ and $\theta(\cup K)=\bigcup \theta(K)$, where $\theta(K)$ is the image of the set $K$ under the mapping $\theta$. If there exists a complete homomorphism of $L$ onto $L^{\prime}$, then $L^{\prime}$ is called a complete homomorphic image of $L$.

If $\phi=\left\{\phi_{a} \mid a \in A\right\}$ is an indexed family of subsets of $L$ with indexing set $A$, let $S(\phi)$ denote the set of mappings $s$ of $A$ into $L$ such that for every $a \in A, s(a) \in \phi_{a}$. If $s$ is a mapping of $A$ into $L$, let $s(A)$ denote the image of the set $A$ under the mapping $s$.

Definition 3. Let $L$ be a complete lattice. Meets are completely distributive with respect to joins in $L$ if for every indexed family $\phi$ of subsets of $L$ with indexing set $A$,

$$
\cap\left\{\bigcup_{\phi_{a}} \mid a \in A\right\}=\bigcup\left\{\cap_{s}(A) \mid s \in S(\phi)\right\} .
$$

Joins are completely distributive with respect to meets in $L$ if for every indexed family $\phi$ of subsets of $L$ with indexing set $A$,

$$
\cup\left\{\cap_{\phi_{a}} \mid a \in A\right\}=\bigcap\left\{\bigcup_{s}(A) \mid s \in S(\phi)\right\} .
$$

$L$ is called completely distributive if both these conditions are satisfied.

If $L$ is any partially ordered set and if $x \in L$, then let $P(x)$ denote the set of $t \in L$ such that $t \leqq x$.

Definition 4. Let $L$ be any partially ordered set. A subset $M$ of $L$ is called a semi-ideal of $L$ if, for every $x \in M, P(x) \subset M$.

Presented to the Society, June 16, 1951; received by the editors January 7, 1952.

${ }^{1}$ See G. Birkhoff, Lattice theory, rev. ed., Amer. Math. Soc. Colloquium Publications, vol. 25, 1948, p. 147, Problem 69, and p. 166. References to the work of Tarski are given here. 
Definition 5. Let $L$ be a complete lattice. An element $x$ of $L$ is called completely join-irreducible if for every $K \subset L$ such that $x \leqq U K$ there exists a $y \in K$ such that $x \leqq y$.

2. Statements of the theorems. The principal theorem of this paper is the following representation theorem for completely distributive complete lattices.

THEOREM 1. In a complete lattice $L$ each of the following conditions implies the others:

(I) $L$ is completely distributive;

(II) Meets are completely distributive with respect to joins in $L$;

(III) Joins are completely distributive with respect to meets in L;

(IV) $L$ is a complete homomorphic image of a complete ring of sets.

An example will be given to show that the word "homomorphic" in condition (IV) cannot be replaced by the word "isomorphic." In the discussion of this example, the following theorem is useful.

THEOREM 2. For a complete lattice $L$ to be isomorphic with a complete ring of sets the following condition is necessary and sufficient:

(*) Every element of $L$ is the join of a set of completely join-irreducible elements.

3. Proof of Theorem 1. The following lemmas are used in the proof of Theorem 1. Proofs are not given for those which are well known.

LEMMA 1. Every complete ring of sets, partially ordered by set inclusion, is a complete lattice, in which joins are unions and meets are intersections, and this complete lattice is completely distributive.

LEMMA 2. If a complete lattice is a complete homomorphic image of a completely distributive complete lattice, then it is completely distributive.

LEMmA 3. The family of semi-ideals of any partially ordered set is a complete ring of sets.

Lemma 4. Let $L$ be a complete lattice and let $\phi$ be an indexed family of semi-ideals of $L$ with indexing set $A$. Then $\prod \phi=\left\{\bigcap_{s}(A) \mid s \in S(\phi)\right\}$.

Proof. If $x \in \prod \phi$, then for every $a \in A, x \in \phi_{a}$. Let $s_{0}$ be the mapping of $A$ into $L$ such that, for every $a \in A, s_{0}(a)=x$. Then $s_{0}(A)=\{x\}$, and $\bigcap_{s_{0}}(A)=x$. Then $x \in\left\{\bigcap_{s}(A) \mid s \in S(\phi)\right\}$. On the other hand, if $x \in\{\cap s(A) \mid s \in S(\phi)\}$, then there exists a mapping $s_{1}$ of $A$ into $L$ such that $s_{1} \in S(\phi)$ and $x=\bigcap_{1}(A)$. Then, for every $a \in A$, $s_{1}(a) \in \phi_{a}$ and $x \leqq s_{1}(a)$ and $\phi_{a}$ is a semi-ideal of $L$. Therefore, for 
every $a \in A, x \in \phi_{a}$; that is, $x \in \prod \phi$.

Lemma 5. Let $L$ be a complete lattice and let $R(L)$ be the complete lattice of semi-ideals of $L$. Let $\theta$ be the mapping of $R(L)$ into $L$ defined as follows: if $M \in R(L)$, then $\theta(M)=\cup M$. For $\theta$ to be a complete homomorphism of $R(L)$ onto $L$ it is necessary and sufficient that $L$ satisfy condition (II).

Proof. $R(L)$ is a complete ring of sets, by Lemma 3. Partially ordered by set inclusion, $R(L)$ is a completely distributive complete lattice, by Lemma 1 . If $\theta$ is a complete homomorphism of $R(L)$ onto $L$, then $L$ is completely distributive, by Lemma 2 . Hence it is necessary that $L$ satisfies condition (II).

To prove sufficiency, let $\phi$ be a subset of $R(L)$. Then $\phi$ is a family of semi-ideals of $L ; \bigcup_{\phi}=\sum \phi$ and $\bigcap_{\phi}=\prod \phi$. There is no loss of generality in supposing that $\phi$ is indexed by a set $A: \phi=\left\{\phi_{a} \mid a \in A\right\}$.

By the definition of $\theta, \theta(\cup \phi)=\theta\left(\sum \phi\right)=\cup \sum \phi$. In any complete lattice $\bigcup \sum \phi=\bigcup\left\{\bigcup \phi_{a} \mid a \in A\right\}$. Now $\bigcup\left\{\bigcup \phi_{a} \mid a \in A\right\}=\bigcup\left\{\theta\left(\phi_{a}\right) \mid a \in A\right\}$ $=\bigcup \theta(\phi)$. Therefore $\theta(U \phi)=\bigcup \theta(\phi)$; that is, the mapping $\theta$ preserves joins.

By the definition of $\theta, \theta\left(\bigcap_{\phi}\right)=\theta\left(\prod \phi\right)=\cup \prod \phi$. By Lemma 4, П $\phi$ $=\left\{\bigcap_{s}(A) \mid s \in S(\phi)\right\}$. Therefore U $\bigcup \phi=\cup\left\{\cap_{s}(A) \mid s \in S(\phi)\right\}$. If $L$ satisfies condition (II), then $U\{\cap s(A) \mid s \in S(\phi)\}=\bigcap\left\{\cup_{\phi_{a}} \mid a \in A\right\}$. Now $\cap\left\{\bigcup_{\phi_{a}} \mid a \in A\right\}=\bigcap\left\{\theta\left(\phi_{a}\right) \mid a \in A\right\}=\cap \theta(\phi)$. Therefore, $\theta\left(\bigcap_{\phi}\right)$ $=\cap \theta(\phi)$; that is, the mapping $\theta$ preserves meets.

For every $x \in L, P(x) \in R(L)$ and $\theta(P(x))=\cup P(x)=x$. Therefore $\theta$ is a mapping of $R(L)$ onto $L$. This completes the proof of Lemma 5.

It follows from Lemmas 5 and 3 that condition (II) implies condition (IV). By Lemmas 1 and 2, condition (IV) implies condition (I). By Definition 3, condition (I) implies condition (III). Hence condition (II) implies condition (III). The same argument applied to the dual lattice would show that condition (III) implies condition (II). This completes the proof of Theorem 1.

4. Proof of Theorem 2. Let $R$ be a complete ring of subsets of a set $X$. For every $p \in X$, let $F(p)$ be the intersection of the family of sets in $R$ which have $p$ as a member. Then, for every $p \in X, F(p) \in R$ and $p \in F(p)$. If $G \subset R$ and $F(p) \subset \sum G$, then $p \in \sum G$. Then there exists a $T \in G$ such that $p \in T$. Then $F(p) \subset T$. Therefore $F(p)$ is completely join-irreducible.

Let $T \in R$. If $q \in T$, then $q \in \sum\{F(p) \mid p \in T\}$, since $q \in F(q)$. On the other hand, if $q \in \sum\{F(p) \mid p \in T\}$, then for some $p \in T, q \in F(p)$. 
Then $F(p) \subset T$ and $q \in T$. Therefore $T=\sum\{F(p) \mid p \in T\}$. Thus the condition $\left({ }^{*}\right)$ is necessary.

To prove the sufficiency of condition $\left({ }^{*}\right)$, let $X$ be the set of completely join-irreducible elements of $L$, and for every $x \in L$ let $M(x)$ be the set of $t \in X$ such that $t \leqq x$. Condition $\left({ }^{*}\right)$ implies that $x=\bigcup M(x)$ for every $x \in L$, whence it follows that $x \leqq y$ if and only if $M(x) \subset M(y)$. Let $C(L)$ be the family of all sets $M(x)$. If $C(L)$ is partially ordered by set inclusion, then $C(L)$ is isomorphic with $L$.

If $K \subset L$, then $\prod\{M(x) \mid x \in K\}=M(\cap K)$. For $p \in \prod\{M(x) \mid x$ $\in K\}$ if and only if, for every $x \in K, p \leqq x$ and $p \in X$. An equivalent condition is that $p \leqq \cap K$ and $p \in X$; in other words, that $p \in M(\cap K)$.

If $K \subset L$, then $\sum\{M(x) \mid x \in K\}=M(\cup K)$. For if $p \in \sum\{M(x) \mid x$ $\in K\}$, then $p \in M\left(x_{0}\right)$ for some $x_{0} \in K$. Then $p \in X$ and $p \leqq x_{0}$. Therefore $p \leqq \bigcup K$ and $p \in M(\cup K)$. On the other hand, if $p \in M(\cup K)$, then $p \in X$ and $p \leqq \cup K$. Since $p \in X, p$ is completely join-irreducible; hence there exists a $y \in K$ such that $p \leqq y$. Then $p \in M(y)$, so that $p \in \sum\{M(x) \mid x \in K\}$.

This shows that $C(L)$ is a complete ring of subsets of the set $X$, and the proof of Theorem 2 is complete.

5. An example. The set $U$ of real numbers $x$ such that $0 \leqq x \leqq 1$, ordered in the natural way, is a complete lattice. Joins are least upper bounds and meets are greatest lower bounds, with the specification that the join of the empty set is 0 and the meet of the empty set is 1 . This complete lattice is easily seen to be completely distributive.

No element of $U$ is completely join-irreducible, since every element $x \in U$ is the join of the set of $y \in U$ such that $y<x$. It follows that if $x \in U$ and $x \neq 0$, then $x$ is not the join of a set of completely joinirreducible elements. Therefore, $U$ is not isomorphic with a complete ring of sets.

For every $x \in U$ there are exactly two semi-ideals of $U$ whose join is $x$. One of these is the set of $y \in U$ such that $y \leqq x$; the other is the set of $y \in U$ such that $y<x$. The mapping which takes each semiideal into its join is a (2-1) mapping of the complete ring of semiideals of $U$ onto $U$, and it is a complete homomorphism.

Columbia University 